Article

\title{
Use of Indocyanine Green (ICG), a Medical Near Infrared Dye, for Enhanced Fluorescent Imaging-Comparison of Organic Anion Transporting Polypeptide 1B3 (OATP1B3) and Sodium-Taurocholate Cotransporting Polypeptide (NTCP) Reporter Genes
}

\author{
Menq-Rong $\mathrm{Wu}^{1,2}{ }^{1}$, Yi-You Huang ${ }^{1}$ and Jong-Kai Hsiao ${ }^{2,3, *(1)}$ \\ 1 Institute of Biomedical Engineering, National Taiwan University, No. 1, Sec. 4, Roosevelt Rd., Taipei 10617, \\ Taiwan; nicole750707@gmail.com (M.-R.W.); yyhuang@ntu.edu.tw (Y.-Y.H.) \\ 2 Department of Medical Imaging, Taipei TzuChi General Hospital, Buddhist Tzu-Chi Medical Foundation, \\ No.289, Jianguo Rd., Xindian Dist., New Taipei city 23142, Taiwan \\ 3 School of Medicine, Tzu Chi University, No. 701, Sec. 3, Zhongyang Rd. Hualien 97004, Taiwan \\ * Correspondence: jongkai@tzuchi.com.tw; Tel.: +886-2-6628-9779-61114
}

Academic Editor: Maged Henary

Received: 27 May 2019; Accepted: 20 June 2019; Published: 21 June 2019

\begin{abstract}
Molecular and cellular imaging in living organisms have ushered in an era of comprehensive understanding of intracellular and intercellular events. Currently, more efforts have been focused on the infrared fluorescent dyes that facilitate deeper tissue visualization. Both sodium taurocholate cotransporting polypeptide (NTCP) and organic-anion-transporting polypeptide 1B3 (OATP1B3) are capable of carrying indocyanine green (ICG) into the cytoplasm. We compared the feasibility of NTCP and $O A T P 1 B 3$ as reporter genes in combination with ICG. NTCP and OATP1B3 were transduced into HT-29 cells. Genetically modified HT-29 cells were inoculated into nude mice. ICG was administered in vitro and in vivo and the signals were observed under confocal microscopy, flow cytometry, multimode microplate reader, and an in vivo imaging system. Both NTCP- and OATP1B3-expressing cells and xenografts had higher ICG intensities. The OATP1B3-expressing xenograft has a higher ICG uptake than the NTCP-expressing xenograft. NTCP or OATP1B3 combined with ICG could serve as a noninvasive imaging modality for molecular and cellular imaging. OATP1B3 outperforms NTCP in terms of in vivo imaging.
\end{abstract}

Keywords: sodium taurocholate cotransporting polypeptide (NTCP); organic-anion-transporting polypeptide 1B3 (OATP1B3); indocyanine green (ICG); in vivo imaging system (IVIS)

\section{Introduction}

Molecular and cellular imaging in living organisms has ushered in an era of comprehensive understanding of intracellular and intercellular events. Labeling fluorescent tags or nanoparticles that exhibit magnetic resonance (MR)/computed tomography (CT)/positron emission tomography (PET) signals is convenient for short-term cell tracking. However, a dilutional effect exists because of cell division and metabolism [1,2]. Therefore, genetic modification with reporter genes is a superior approach for long-term repeated cell tracking. Currently, numerous reporter genes are applied in molecular imaging activities, such as traditional green fluorescent protein (GFP), red fluorescent protein (RFP), and bioluminescence imaging (BLI) [3,4]. Examples of reporter genes include herpes simplex virus type 1 thymidine kinase reporter and dopamine transporter in PET [5,6]; and transferrin receptor, 
organic-anion-transporting polypeptide 1B1 (OATP1B1), and organic-anion-transporting polypeptide 1B3 (OATP1B3) in magnetic resonance imaging (MRI) [7-9]. Fluorescence and BLI are advantageous based on their sensitivity compared with other imaging modalities. However, the penetration path depth is limited due to the nature of optics. Currently, more efforts are being focused on infrared fluorescent dyes that facilitate much deeper tissue visualization. Indocyanine green (ICG), one of the best candidates, has been used extensively in clinical practice [10-13]. The excitation/emission wavelength of ICG is $760 / 830 \mathrm{~nm}$, facilitating superior tissue penetration compared with conventional near-infrared (NIRF) dyes such as Cy5.5 (excitation/emission wavelength of $675 \mathrm{~nm} / 694 \mathrm{~nm}$ ) [14-16].

Both sodium taurocholate cotransporting polypeptide (NTCP) and OATP1B3 are capable of carrying ICG into the cytoplasm [17]. OATP1B3 belongs to the solute carrier organic anion transporter family (OATP family) and is expressed in hepatocytes. It is a transmembrane glycoprotein responsible for transporting bilirubin, nutrients, drugs, specific types of MRI agents such as gadolinium-ethoxybenzyldiethylenetriaminepentaacetic acid (Gd-EOB-DTPA), and ICG $[17,18]$. NTCP, which exhibits similarity with OATP1B3, is a sodium-dependent transporter that belongs to the solute carrier family of transporters (SLC10). It transports bile salts, sulfated compounds, thyroid hormones, drugs, toxins, and Gd-EOB-DTPA [18,19]. NTCP and OATPs are responsible for the transfer of bile salts from plasma into the liver [20]. Moreover, recent studies have reported a novel characteristic of NTCP as an entry transporter of hepatitis B virus (HBV) and hepatitis D virus (HDV) [21].

We have previously reported the efficacy of OATP1B3 combined with ICG as a reporter gene/fluorescent source imaging system that is capable of imaging OATP1B3 activity in a HT-1080 tumor-bearing nude mouse for up to $96 \mathrm{~h}$, a long period compared to other methods. ICG has a fast-binding property with plasma proteins, enters cells through transporters such as OATP1B3 and NTCP, and is excreted by the liver into bile juice rapidly $[17,22]$. We have also investigated NTCP as a reporter for monitoring the treatment of HBV infections [23]. However, the feasibility of manipulating NTCP as a reporter is yet to be compared with OATP1B3 in combination with the ICG imaging system. The goal of the study was to compare the reporter system combined with ICG in NTCP and OATP1B3.

\section{Results}

\subsection{Validation of NTCP/OATP1B3 Overexpression}

A HT-29 cell line was infected with NTCP- or OATP1B3-expressing lentivirus to generate NTCPor OATP1B3-expressing HT-29 cells. To determine the transduction efficiency, we evaluated the protein levels of NCTP or OATP1B3 in the cells using Western blotting. NTCP- or OATP1B3-overexpressing HT-29 cells carried more NTCP or OATP1B3 (Figure S1a,b). The NTCP and OATP1B3 in the cells were colocalized with phalloidin, indicating that they were mainly in the cellular membrane (Figure S1c,d). Moreover, NTCP was also expressed in the cytoplasm and the nucleus (Figure S1c). In addition, cell viability did not change following transduction (Figure S2).

\subsection{Evaluation of ICG Transporting Capacity in NTCP and OATP1B3 In Vitro}

After treatment of the control, and NTCP- and OATP1B3-expressing HT-29 cells with ICG, NTCP-expressing HT-29 cells had a stronger ICG signal than OATP1B3-expressing HT-29 cells (Figure 1). The intensity ratio of ICG observed under flow cytometry was approximately 1:2:5 in the control, OATP1B3-, and NTCP-expressing HT-29 cells (Figure 1a). To assess the detection limits of the in vivo imaging system (IVIS), we determined series cell numbers following ICG treatment. The minimum cells that could be detected were $3.13 \times 10^{3}$ and $1.25 \times 10^{4}$ in NTCP- and OATP1B3-expressing HT-29 cells, respectively (Figure $1 b$ ). In addition, the ICG incubation time was positively related to the ICG intake capacity in NTCP- and OATP1B3-expressing HT-29 cells (Figure 2a). To observe the retention capacity of ICG in the control and in the NTCP- and OATP1B3-expressing HT-29 cells, we detected the ICG signals at different times after ICG treatment. In the beginning, the ICG intensity in NTCP-expressing HT-29 cells was approximately two-fold the ICG intensity of OATP1B3-expressing HT-29 cells. With time, the ICG 
intensity in NTCP-expressing HT-29 cells dropped to levels lower than in OATP1B3-expressing HT-29 cells. Therefore, $72 \mathrm{~h}$ after ICG treatment, ICG intensity in the OATP1B3-expressing HT-29 cells was two times greater than the ICG intensity in NCTP-expressing HT-29 cells (Figure 2b).

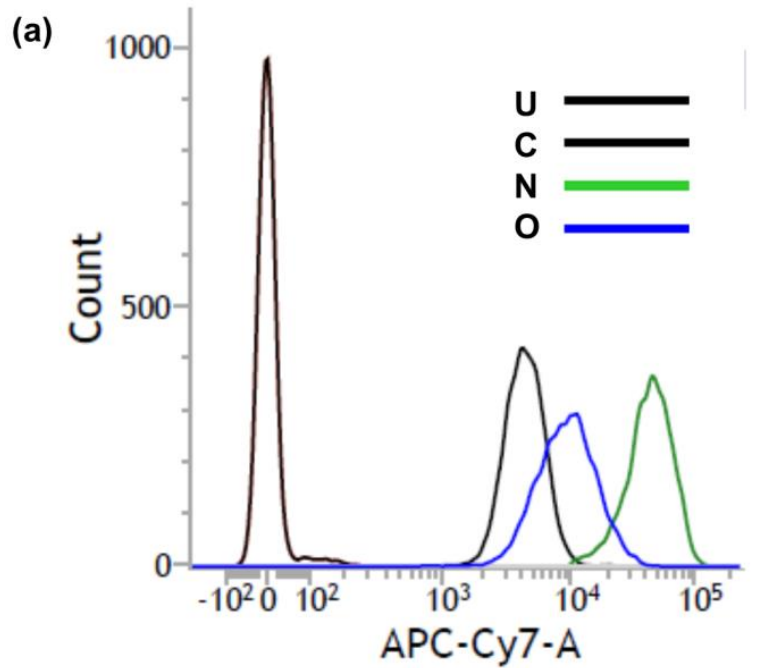

(c)

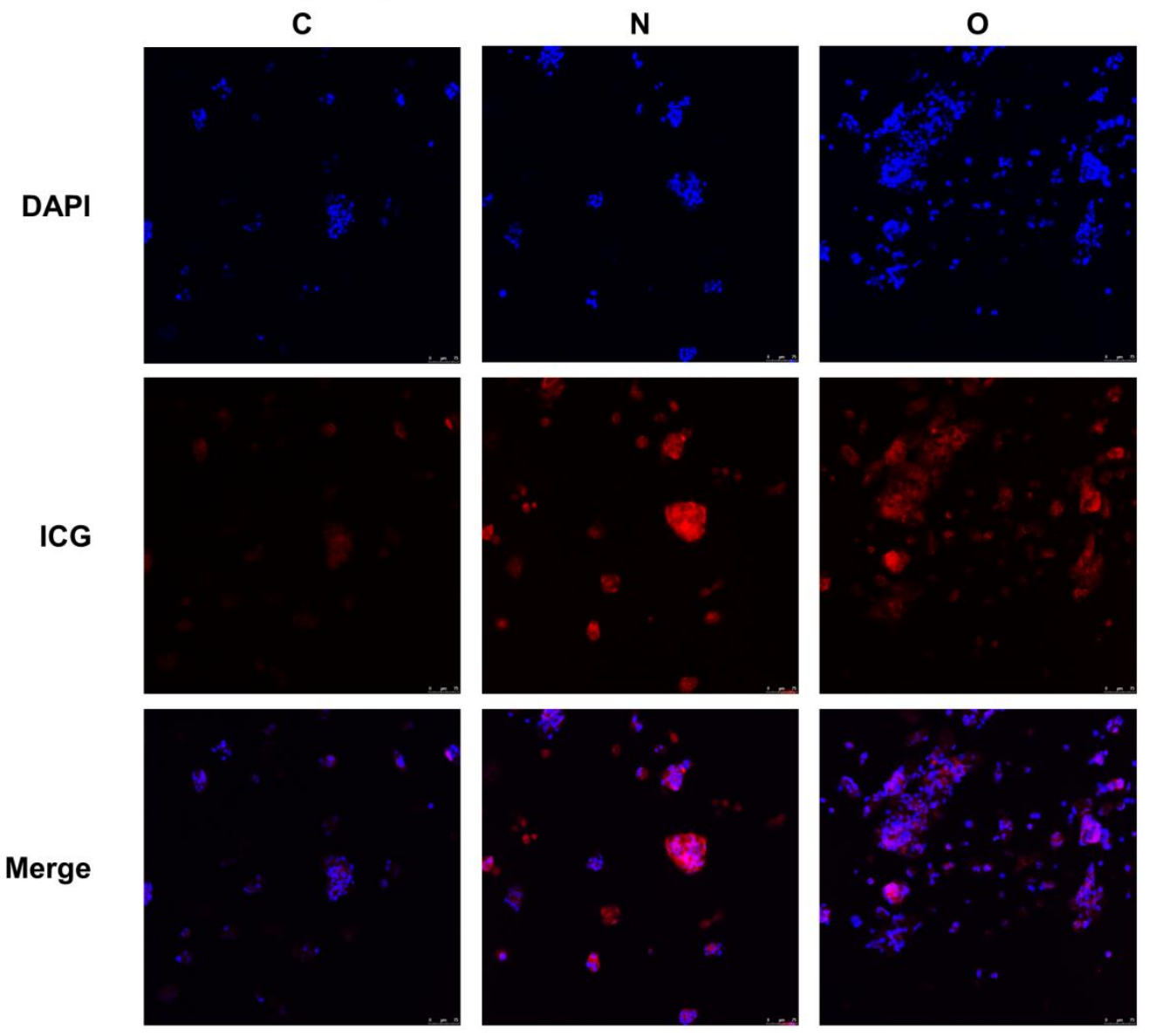

Figure 1. Indocyanine green (ICG) intake capacity in sodium taurocholate cotransporting polypeptide (NTCP)-expressing and organic-anion-transporting polypeptide 1B3 (OATP1B3)-expressing HT-29 cells. (a) The cells were measured by using flow cytometry at the APC-Cy7 wavelength $(785 \mathrm{~nm})$ to observe ICG intensity. (b) The diluted series cells were detected using an in vivo imaging system (IVIS). (c) One hour after ICG was added to the control and the NTCP- and OATP1B3-expressing HT-29 cells, the cells were monitored under a confocal microscope. C: control HT-29. U: untreated. N: NTCP-expressing HT-29 cells. O: OATP1B3-expressing HT-29 cells.

(b)

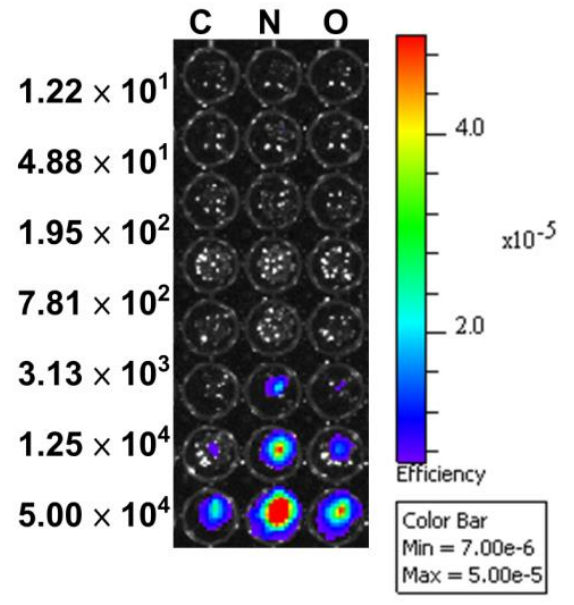


(a)

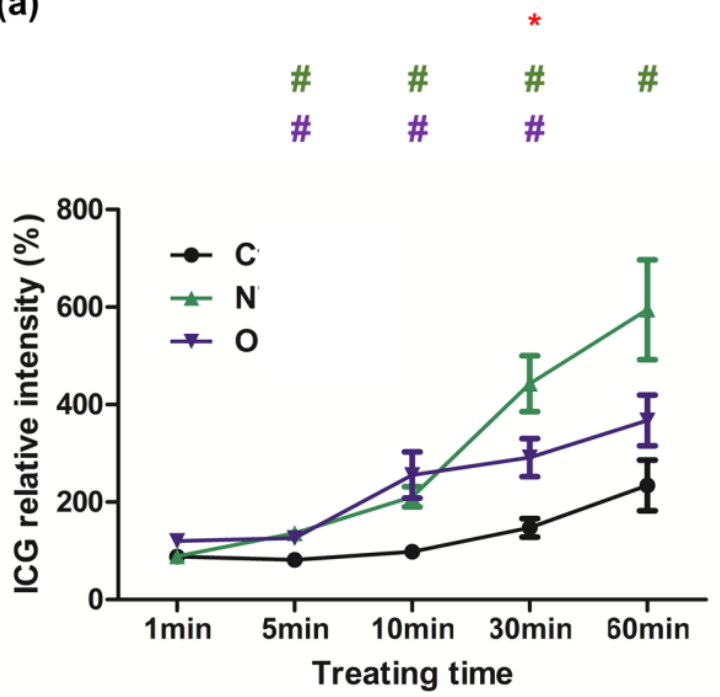

(b)

$\begin{array}{lllllll}\# & & & * & \# & * & * \\ \# & \# & \# & \# & \# & * & \# \\ \# & \# & \# & \# & \# & \# & \#\end{array}$

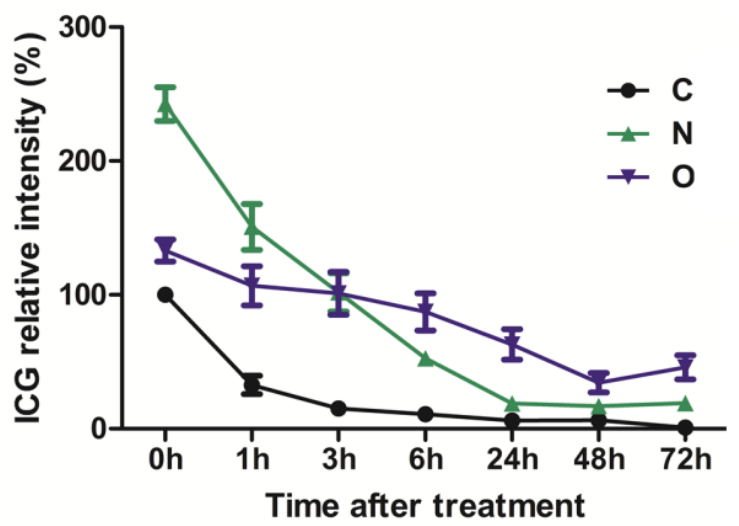

Figure 2. ICG intake and retention in NTCP- and OATP1B3-expressing HT-29 cells. (a) ICG intensity was detected after ICG treatment for 1,5, 10, 30, and $60 \mathrm{~min}$ in the control and the NTCP-and OATP1B3-expressing HT-29 cells. $\mathrm{N}=3$. (b) ICG intensity was detected at $0,1,3,6,24,48$, and $72 \mathrm{~h}$ after ICG treatment for $1 \mathrm{~h}$ in the control and NTCP- and OATP1B3-expressing HT-29 cells. $\mathrm{N}=3$. C: control HT-29 cells. N: NTCP-expressing HT-29 cells. O: OATP1B3-expressing HT-29 cells. Error bars represent the standard error of the mean (SEM). ${ }^{*} p<0.05,{ }^{\#} p<0.01 .{ }^{*}$ in purple color indicates control compared with OATP1B3-expressing HT-29 cells. * and * in green color indicate control compared with NTCP-expressing HT-29 cells. * and * in red color indicate NTCP-expressing HT-29 cells compared with OATP1B3-expressing HT-29 cells.

\subsection{Evaluation of ICG Transporting Ability between NTCP and OATP1B3 In Vivo}

After nude mice were administrated with the control and NTCP/OATP1B3-expressing HT-29 cells on each side near the hind limb, we administered ICG intraperitoneally to track the ICG signal in the following days. Both NTCP- and OATP1B3-expressing tumor cells had higher ICG signals than the control tumor cells. In addition, the OATP1B3-expressing tumor cells had higher ICG signals than the NTCP-expressing tumor cells (Figure 3a).

The OATP1B3-expressing tumor cells had the highest ICG intensity followed by the NTCP-expressing tumor cells and the control tumor cells (Figure $3 \mathrm{~b}$ ). To obtain an enhanced intensity ratio in NTCP- and OATP1B3-expressing tumor cells, we compared their normalized ICG intensities with the ICG intensity of control tumor cells. After normalization was completed, ICG intensity in the NTCP-expressing tumor cells was approximately 1.5 times that of the control tumor cells at all time points. In addition, ICG intensity in OATP1B3-expressing tumor cells was two times higher at $4 \mathrm{~h}$ and approximately 4 times higher at 24, 48, and $72 \mathrm{~h}$ compared to the ICG intensity of control tumor cells (Figure 3c). 

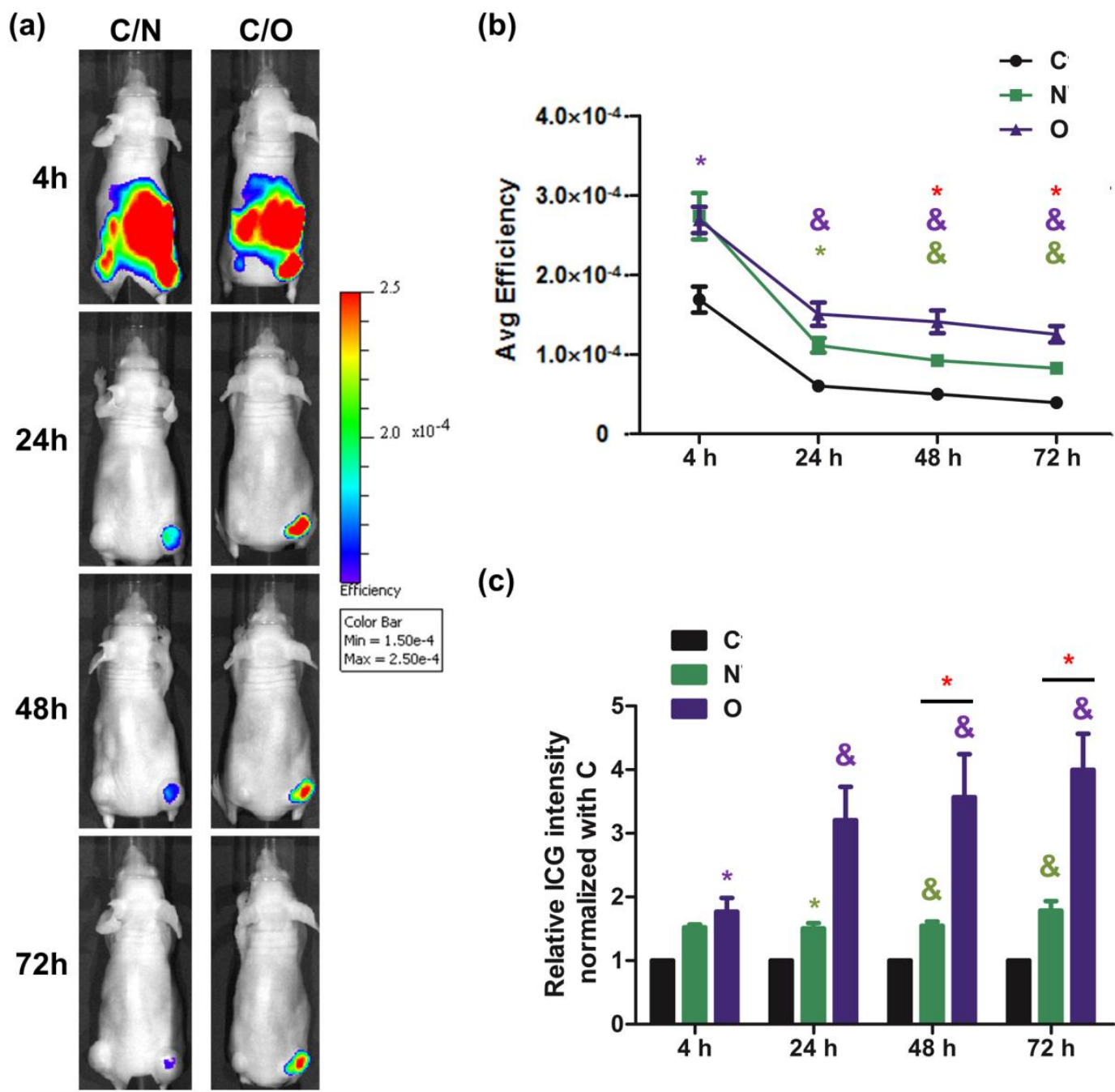

(c)

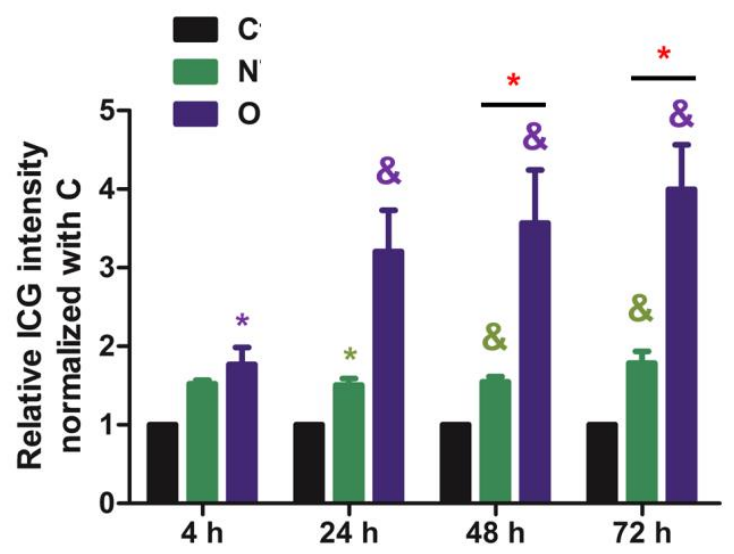

Figure 3. Evaluation of ICG intake in vivo. (a) The ICG signal was observed using IVIS at 4, 24, 48, and $72 \mathrm{~h}$ after injecting ICG to NTCP- and OATP1B3-expressing tumor-bearing mice. The tumor on the left hind leg was a controlled tumor, and on the right was an NTCP-expressing tumor at the top and an OATP1B3-expressing tumor at the bottom. (b) The relationship between tumor size and the total efficiency of ICG at $48 \mathrm{~h}$ after injecting ICG to NTCP- and OATP1B3-expressing tumor-bearing mice. (c) After the ICG signal was normalized based on control tumor size to obtain relative ICG intensity, we compared the relative ICG intensities in the control and NTCP- and OATP1B3-expressing tumor cells at 4, 24, 48, and $72 \mathrm{~h}$ after injecting ICG into the NTCP- and OATP1B3-expressing tumor-bearing mice. $\mathrm{N}=3$ and $\mathrm{N}=4$ for NTCP and OATP1B3 tumor-bearing mice, respectively. C: control tumor. $\mathrm{N}$ : NTCP-expressing tumor. O: OATP1B3-expressing tumor. Error bars indicate the SEM. ${ }^{*} p<0.05$, ${ }^{\#} p<0.01, \& p<0.001 .{ }^{*}, \#, \&$ in purple and green color indicate the control compared with OATP1B3and NTCP-expressing tumor cells, respectively. ${ }^{*}$ in red color indicates the NTCP-expressing tumor cells compared with OATP1B3-expressing tumor cells. Ctrl: control tumor cells.

\subsection{Assessment of Gd-EOB-DTPA Intake In Vivo in MRI}

OATP1B3 and NTCP are both Gd-EOB-DTPA transporters [18]; therefore, they could be considered dual reporter proteins. However, MRI revealed that NCTP-expressing cells did not exhibit the Gd-EOB-DTPA signal in vitro (Figure 4a). In the IVIS modality, both NTCP- and OATP1B3-expressing tumor cells had slightly elevated ICG signals (Figure 4c). In addition, OATP1B3-expressing tumor cells had higher MR imaging signals (Figure 4c). The MR signal intensity was approximately two times the intensity in OATP1B3-expressing tumor cells compared with the control and NTCP-expressing tumor cells (Figure 4b). Moreover, tumor size was not a factor influencing MRI signal intensity (Figure 4d). 
(a)

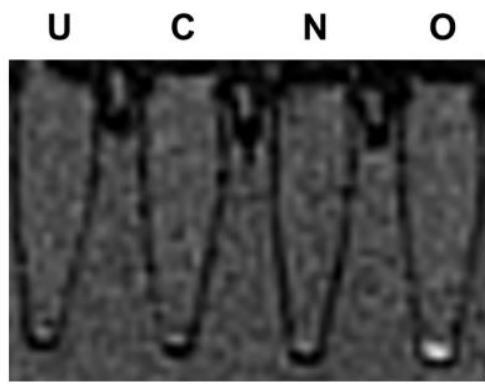

(c)

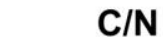

$\mathrm{C} / \mathrm{N}$

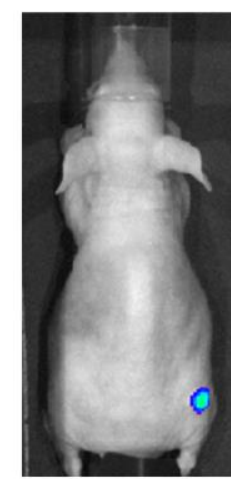

11

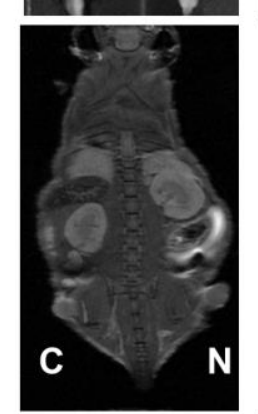

C/O
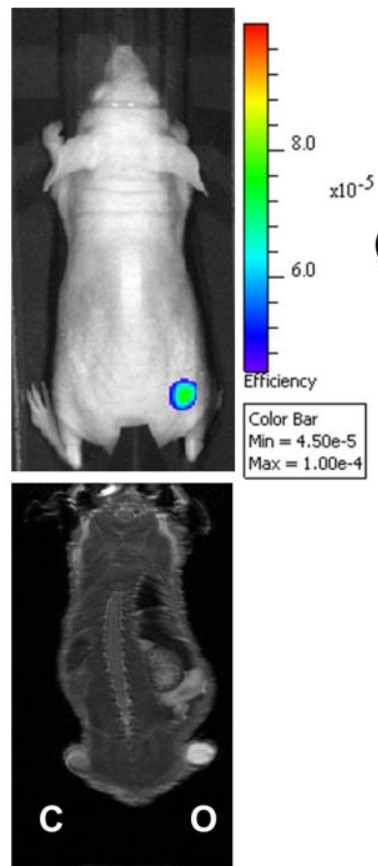

(b)

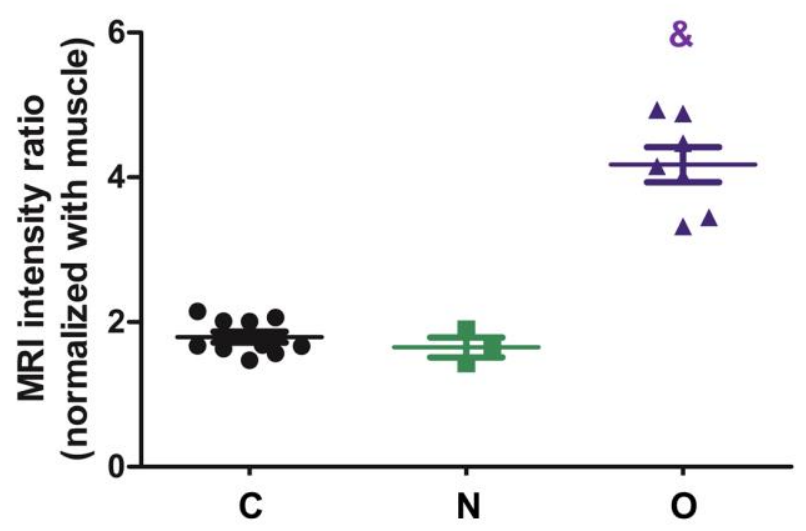

(d)

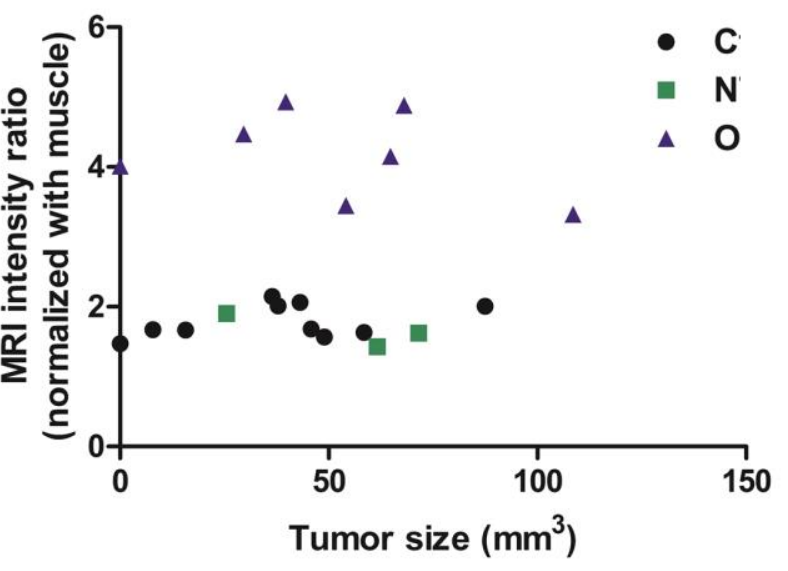

Figure 4. MRI contrast-Gd-EOB-DTPA intake in vivo in magnetic resonance imaging (MRI). (a) After HT-29 cells were transduced into NTCP and OAT1B3 independently, treated with Gd-EOB-DTPA (MRI contrast) for $4 \mathrm{~h}$, and detected using 3T-MRI scanning. (b) The relative signal intensity of the control and NTCP- and OATP1B3-expressing xenografts adjusted based on the signal intensity of muscles in MR images. Error bars indicate the SEM. $N=8, N=3$, and $N=5$ in control, $N T C P$-expressing tumor cells, and OATP1B3-expressing tumor cells, respectively. (c) ICG intensity was acquired using IVIS $9 \mathrm{~d}$ after xenograft. MRI intensity was detected using 7T-MRI approximately $1 \mathrm{~h}$ after injecting MRI contrast into NTCP- and OATP1B3-expressing tumor-bearing mice $11 \mathrm{~d}$ after xenografting. The tumor on the left hind leg was the control tumor, and on the right was the NTCP-expressing tumor (at the top) and the OATP1B3-expressing tumor (at the bottom). (d) The relationship between tumor size and MRI signal at $1 \mathrm{~h}$ after ICG injection into NTCP- and OATP1B3-expressing tumor-bearing mice. C: control HT-29 cells or tumor. N: NTCP-expressing HT-29 cells or tumor. O: OATP1B3-expressing HT-29 cells or tumor. \& $p<0.001$.

\subsection{Evaluation of ICG Transporting Capacity between NTCP and OATP1B3 In Vivo}

At $2 \mathrm{~d}$ after ICG administration, the NTCP- or OATP1B3-expressing tumor-bearing mice were sacrificed to observe the biodistribution of ICG through IVIS. In the NTCP- and OATP1B3-expressing tumor-bearing mice, OATP1B3-expressing tumor cells contained considerable amounts of ICG, followed by the liver, NTCP-expressing tumor, and the control tumor. Other organs had minimal amounts of ICG (Figure 5a,b). In addition, NTCP and OATP1B3 proteins were mainly expressed in NTCP- and OATP1B3-expressing tumors, respectively (Figure 5c,d). 
(a)

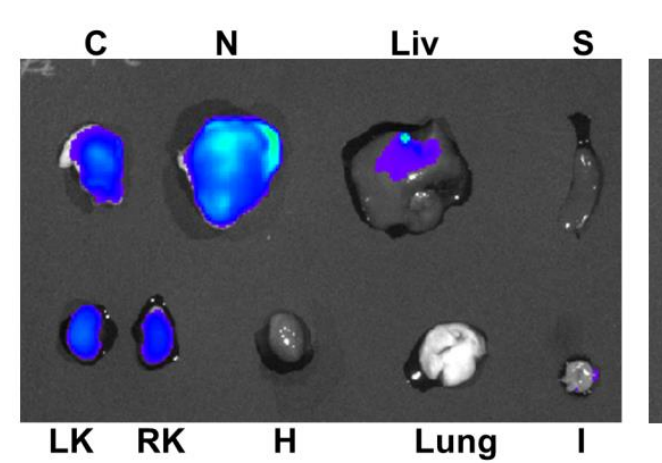

(b)

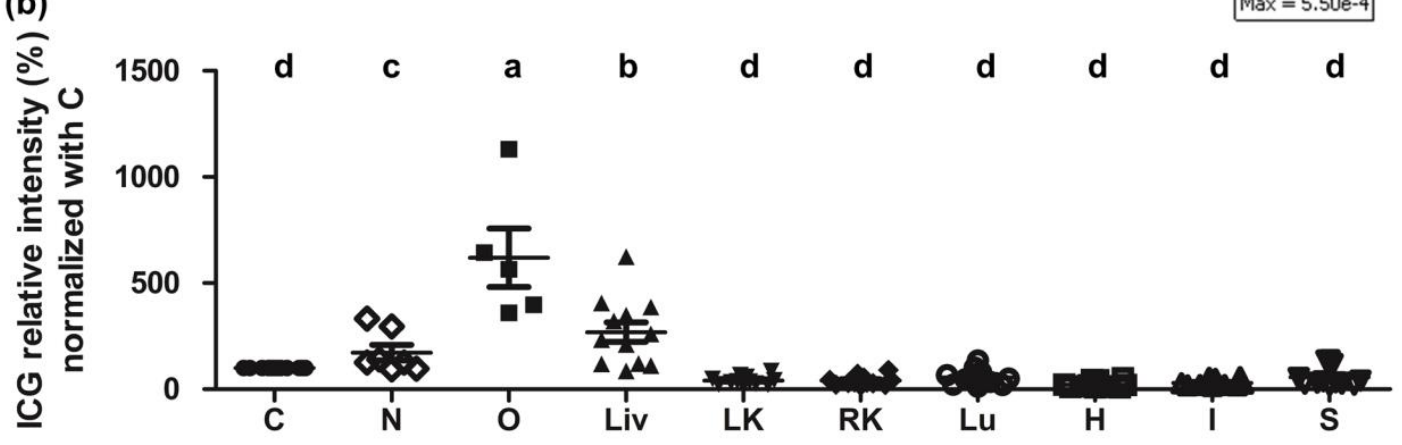

(c)

(d)
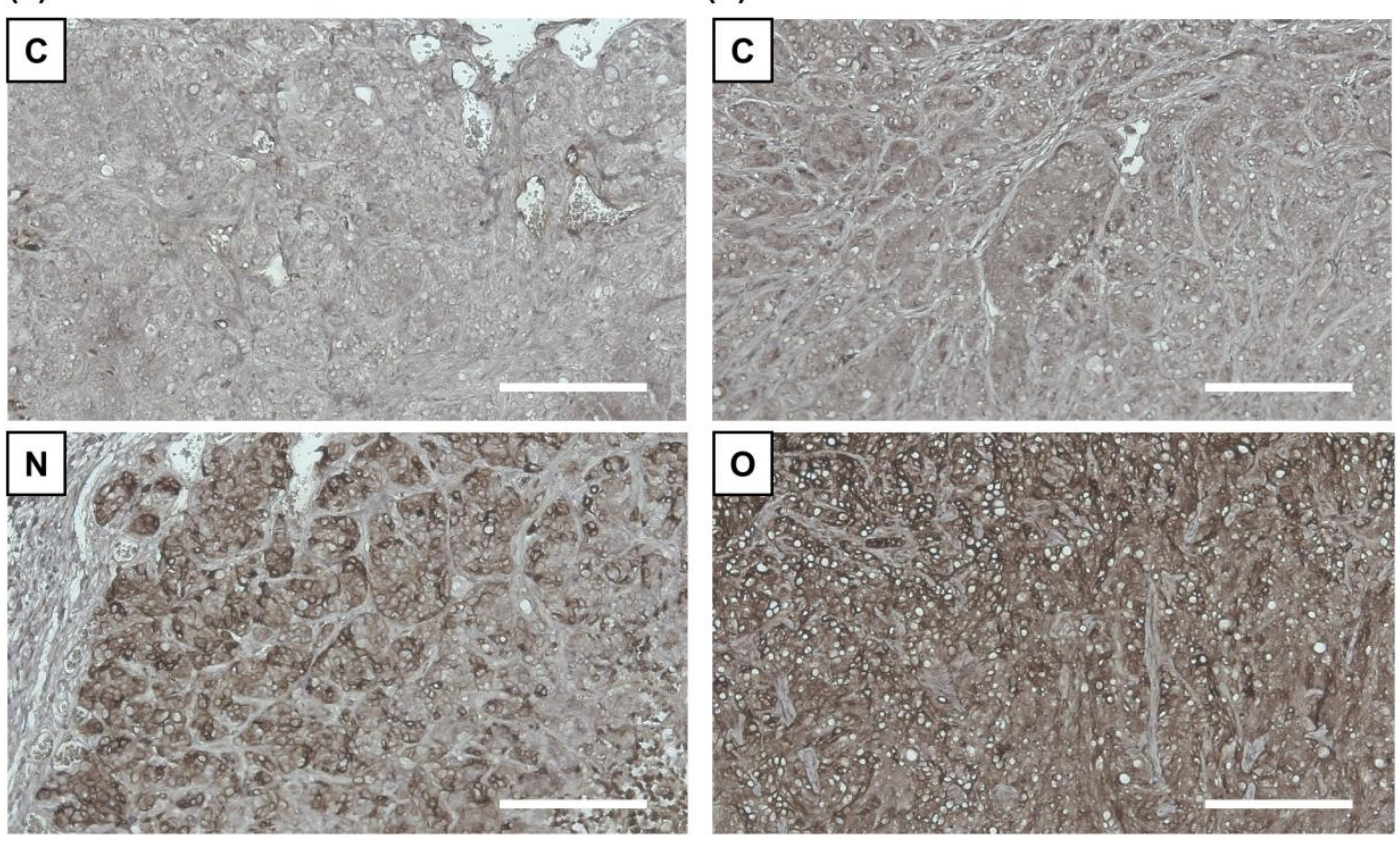

Figure 5. ICG intake ex vivo in the IVIS. After the administration of ICG for $2 \mathrm{~d}$, we sacrificed the mice to evaluate the ICG signals using IVIS. (a) Ex vivo IVIS of NTCP- and OATP1B3-expressing tumor-bearing mice. C: control tumor; N: NTCP-expressing tumor; O: OATP1B3-expressing tumor. Liv: liver; LK: left kidney; RK: right kidney; Lu: Lung; H: heart; S: spleen; I: small intestine. (b) Quantification of ex vivo IVIS data from NTCP- and OATP1B3-expressing tumor-bearing mice; $\mathrm{N}=7$ and $\mathrm{N}=5$, respectively. Error bars indicate the SEM. a, b, c, and d indicate statistically significant differences among groups. (c) Tumors stripped from control and NTCP-expressing tumor-bearing mice stained for NTCP. (d) Tumors stripped from control and OATP1B3-expressing tumor-bearing mice stained for NTCP. The nuclei were stained using hematoxylin. Scale bar: $100 \mu \mathrm{m}$. 


\section{Discussion}

The experiments described in the present study illustrate novel reporter genes that present a novel imaging modality. Both NTCP and OATP1B3 combined with ICG could be applied in near infrared (NIR) imaging modalities such as IVIS and photoacoustic imaging. In addition, OATP1B3 could serve as an MR reporter gene for MRI applications.

Our results showed that NTCP and OATP1B3 are safe reporter genes because NTCP and OATP1B3 transduction did not influence cell viability and tumor size (Figures S2 and S3). In addition, ICG is a Food and Drug Administration (FDA)-approved NIR compound in which safety margins have been explored extensively. It maintains cell viability, which minimizes potential side effects in the course of drug-drug interaction experiments. The characteristics of NIR and their accessibility in laboratories make cell monitoring using the techniques presented herein achievable.

The single modality of imaging the acquired discrete information limits our capacity to examine tumors comprehensively. Through IVIS combined with MRI, we could obtain more information about tumor growth and tumor status. In addition, we could perform experiments within the minimal animals using noninvasive imaging modalities over relatively short time frames. In our reporter system, we could assess cells using noninvasive imaging modalities at different times in a single animal, which minimized individual error and resources in terms of experiment time and experimental animal costs.

The ICG intake capacity was superior in NTCP-expressing cells to OATP1B3-expressing cells at the beginning, indicating that NTCP had superior ICG transporting capacity. However, with time, NTCP-expressing cells contained smaller amounts of ICG than OATP1B3-expressing cells (Figure 2b). The efflux of ICG is mediated by a multidrug resistance p-glycoprotein (MDR)-3 [24]. No evidence is available to confirm that the overexpression of NTCP enhances the expression of MDR3. Therefore, we deduced that it could be due to the disorientation of the NTCP on the cell membrane. Under normal status, the orientation of NTCP is outside-in. In NTCP-expressing cells, some NTCP could be oriented inside-out so that ICG is pumped out. Therefore, NTCP-expressing cells had lower ICG retention capacity compared with OATP1B3-expressing cells. It has been reported that OATP1B3 and NTCP are Gd-EOB-DTPA transporters (for OATP1B3 $\left[\mathrm{K}_{\mathrm{m}}=4.1 \mathrm{mM}, \mathrm{V}_{\max }=22.7 \mathrm{pmol} / \mathrm{mg} \cdot \mathrm{min}\right]$, and for NTCP $\left[\mathrm{K}_{\mathrm{m}}=0.04 \mathrm{mM}, \mathrm{V}_{\max }=1.4 \mathrm{pmol} / \mathrm{mg} \cdot \mathrm{min}\right]$ ) [18]; however, the ingested Gd-EOB-DTPA was in trace amounts that could not be detected using clinical 1.5T MRI (Figure 4). The results demonstrated the varied transportation mechanisms between NTCP and OATP1B3. In addition, OATP1B3-transduced cells could integrate both ICG and EOB-Gd-DTPA, which is ideal for combined IVIS and MRI systems. Therefore, $O A T P 1 B 3$ is a superior reporter gene for cell tracking and tumor monitoring.

At $4 \mathrm{~h}$ after ICG was injected into NTCP/OATP1B3-expressing tumor-bearing mice, the ICG signal was detected in the whole body and accumulated in the NTCP/OATP1B3-expressing tumors at $24 \mathrm{~h}$ after injection (Figure 3a). The background uptake in most tissues was presumed to be low because NTCP and OATP1B3 exhibit restricted tissue expression [25,26].

Sodium fluorescein (NaFluo) and numerous fluorescein derivatives, which have potential application in in vivo imaging probes, have been identified as substrates mediated by OATP1B1 and OATP1B3 [27]. Considering the structural similarity between NaFluo and fluorescein isothiocyanate (FITC), we tested whether FITC could be applied as an in vivo tracking probe. OATP1B3-expressing HT-29 could assimilate more FITC, as revealed using flow cytometry (Figure S4a); however, it may not have the ability to be applied in in vivo imaging (Figure S4b) owing to the nonspecific binding of FITC to the entire tissue. Based on the results of ICG and FITC application as probes in the OATP1B3 reporter system, ICG was a superior probe for in vivo imaging (Figure 3a and Figure S4b). 


\section{Materials and Methods}

\subsection{Cell Line and Culture}

HEK $293 \mathrm{~T}$ and HT-29 cells were cultured in Dulbecco's modified Eagle medium (Thermo Fisher Scientific, Waltham, MA, USA). We added 10\% fetal bovine serum (Biologic Industries, Cromwell, CT, USA), $100 \mathrm{U} / \mathrm{mL}$ penicillin, and $100 \mathrm{mg} / \mathrm{mL}$ streptomycin (Thermo Fisher Scientific). These cells were cultured in a humidified atmosphere incubator containing $5 \% \mathrm{CO}_{2}$ at $37^{\circ} \mathrm{C}$.

\subsection{Vector Construction and Cell Transfection and Transduction}

Virus production was performed according to the method in Nature Protocols [28]. The plasmids were pWPXL-OATP1B3-ires-Puro and pWPXL-NTCP-ires-Puro that we constructed previously [23]. HT-29 cells $\left(2 \times 10^{5}\right)$ were seeded in 6-well plates for $1 \mathrm{~d}$, which were transduced at a multiplicity of infection of 5 . Subsequently, the cells were selected with $2 \mu \mathrm{g} / \mathrm{mL}$ puromycin (Millipore-Sigma, Billerica, MA, USA) for $10 \mathrm{~d}$ at $1 \mathrm{~d}$ after infection.

\subsection{Evaluations on the Cellular Uptake of ICG}

To test the detecting limitation of the IVIS Spectrum imaging system, $2 \times 10^{6}$ cells were treated with $50 \mu \mathrm{g} / \mathrm{mL}$ ICG (Millipore-Sigma, Billerica, MA, USA) for $1 \mathrm{~h}$ after the spray in 6-well plates for 1 day. The excess ICG was then washed out 3 times with $1 \times$ phosphate-buffered saline (PBS). There were series diluted cells in 96-well black plates for detecting the ICG signal via an IVIS Spectrum imaging system (Xenogen, Perkin Elmer, Waltham, MA, USA). All the data were acquired under similar parameters (emission/excitation channel: ICG/ICG; exposure time: 1 min; binning: medium; lens aperture [f/stop]: 2; field of view: $12 \mathrm{~cm}$ ). The images were presented using radiant efficiency units $\left(\mathrm{p} / \mathrm{s} / \mathrm{cm}^{2} / \mathrm{sr}\right) /\left(\mu \mathrm{W} / \mathrm{cm}^{2}\right)$. The quantification of ICG intensity was based on the average efficiency in the region of interest $(\mathrm{ROI})$.

For intake capacity, $1 \times 10^{6}$ cells were treated with $50 \mu \mathrm{g} / \mathrm{mL}$ ICG for $1 \mathrm{~h}$ after the spray in 12 -well plates for 1 day. Cells were trypsinized, neutralized, and then washed 3 times with PBS. The ICG signal was acquired using a FACSCalibur (BD Biosciences, San Jose, CA, USA). The filter used for the detection was an APC-Cy7 channel (785 nm) filter.

To compare the ICG uptake ability, $2 \times 10^{4}$ cells were seeded in 96-well plates for 1 day before treatment with $50 \mu \mathrm{g} / \mathrm{mL}$ ICG for 1, 5, 10, 30, and $60 \mathrm{~min}$. After the excess ICG was washed out 3 times with 1× PBS, cells were detected using Spark 10M (Tecan Trading AG, Männedorf, Switzerland) at 0, 1, $3,6,24,48$, and $72 \mathrm{~h}$ after ICG treatment. In addition, the ICG signal was observed under a TCS SP5 laser-scanning microscope (Leica, Wetzlar, Germany) with a Cy5 filter.

\subsection{Animal Experiments}

We purchased 6 to 8-week-old female BALB/cAnN.Cg-Foxnlnu/CrlNarl nude mice from the National Laboratory Animal Center. The experimental study was approved by the Institutional Animal Use and Care Committee of Taipei Tzu Chi Hospital, Buddhist Tzu Chi Medical Foundation (106-IACUC-004). The maintenance of the mice followed the suggestions of the Guide for the Care and Use of Laboratory Animals (National Institues of Health, NIH).

\subsection{Xenograft}

HT-29 control cells and NTCP-expressing cells $\left(2.5 \times 10^{6}\right)$ were injected subcutaneously to the nude mice on the left and right side, respectively. The cell number used to inoculate control cells and OATP1B3-expressing cells was $1.0 \times 10^{6}$. We measured the tumor sizes using a digital caliper (Shineteh Instruments, Taipei, Taiwan) before fluorescence imaging and MRI were conducted and estimated using the following formula: [(longest length) $\left.\times(\text { shortest })^{2}\right] / 2$. 


\subsection{Fluorescence and Bioluminescence Imaging In Vivo and Ex Vivo}

Tumor-bearing nude mice were administrated $10 \mathrm{mg} / \mathrm{kg}$ ICG (dissolved in $\mathrm{dd}_{2} \mathrm{O}$ ) intraperitoneally and observed using in vivo fluorescence imaging at 4, 24, 48, and $72 \mathrm{~h}$ after injection using an IVIS Spectrum imaging system (Xenogen; Perkin Elmer). The mice were sacrificed to examine the ICG signal intensity in tumors and organs under IVIS after $10 \mathrm{mg} / \mathrm{kg}$ ICG was injected for $2 \mathrm{~d}$. We acquired all images using the same parameters [emission/excitation channel: ICG/ICG; exposure time: auto; binning: medium; lens aperture: 2; field of view: $12 \mathrm{~cm}$ ]. The imaging data acquired from IVIS are quantified in units of radiant efficiency, which means photons per second per square $\mathrm{cm}$ per steradian/microwatts per square $\mathrm{cm}$. The quantification of ICG intensity was based on the average efficiency in the ROI.

\subsection{MRI In Vitro}

Cells $\left(5 \times 10^{5}\right)$ were seeded in 6-well plates overnight, which were treated with $1.25 \mathrm{mM}$ Gd-EOB-DTPA (Bayer Pharma AG, Berlin, Germany) for $4 \mathrm{~h}$. Cells were PBS washed 3 times using trypsinized. After cells were centrifuged at $1200 \mathrm{rpm}$ for $5 \mathrm{~min}$ in $0.2-\mathrm{mL}$ tubes at $4{ }^{\circ} \mathrm{C}$, the cells were analyzed using a clinical 1.5T MRI system (Siemens Magnetom Aera, Erlangen Germany). We used 2-dimensional T1-weighted fast spin-echo pulse sequences (TR/TE $=700 / 20 \mathrm{~ms})$. The scanning slice thickness was $1.0 \mathrm{~mm}$ with a $0.5-\mathrm{mm}$ gap, and the field of view (FOV) was $14 \mathrm{~cm} \times 6 \mathrm{~cm}$. Moreover, the matrix size was $256 \times 112$.

\subsection{MRI In Vivo}

Nude mice underwent xenografting for 11 days. Images were acquired $1 \mathrm{~h}$ after $200 \mu \mathrm{L}$ of $250 \mathrm{mM}$ Gd-EOB-DTPA was administered intravenously. Images were acquired using a 7T-MRI system (Bruker Biospec 70/30, Ettlingen, Germany). Fast spin-echo pulse sequences (FSE) provided by the vendor were used $(\mathrm{TR} / \mathrm{TE}=842 / 8.6404 \mathrm{~ms}$; resolution $=256 \times 256)$. The slice thickness was $0.5 \mathrm{~mm}$. The FOV was $5 \mathrm{~cm} \times 5 \mathrm{~cm}$. The total scanning time was $3 \mathrm{~min}$ and $20 \mathrm{~s}$ (Number of excitation $(\mathrm{NEX})=10$ ). The images were analyzed using RadiAnt DICOM Viewer (64-bit) (Medixant, Poznan, Poland).

\subsection{Western Blot Analysis}

Immunoblotting analysis was performed as described previously [9]. Primary antibodies were against NTCP (1:1000; Thermo Fisher Scientific), $\alpha$-tubulin (1:5000; Merck Millipore, Burlington, MA, USA), OATP8 (1:1000; Santa Cruz Biotechnology, Dallas, TX, USA), and Glyceraldehyde-3-Phosphate Dehydrogenase (GAPDH) (1:5000; Cell Signaling Technology, Danvers, MA, USA) separately at $4{ }^{\circ} \mathrm{C}$ and were then incubated with 1:5000 horseradish peroxidase-conjugated rabbit/mouse anti-IgG for $1 \mathrm{~h}$ at room temperature.

\subsection{Immunofluorescence}

After the cells were fixed in 4\% formaldehyde, they were blocked with 5\% BSA. Next, the cells were incubated with 1:100 rabbit polyclonal anti-OATP8 antibodies (Santa Cruz, CA, US) and 1:100 anti-NTCP antibodies (Thermo Fisher Scientific, Waltham, MA, USA) at $4{ }^{\circ} \mathrm{C}$ overnight. After 4 times washing in PBST, the slides were treated with 488-conjugated goat anti-rabbit IgG antibodies (Thermo Fisher Scientific, Waltham, MA, USA), DAPI, and rhodamine phalloidin (Thermo Fisher Scientific, Waltham, MA, USA) at room temperature for $1 \mathrm{~h}$. All cover slides were visualized using a Fluorescent Cell Imager (ZOE, Bio-Rad, Hercules, CA, USA).

\subsection{Immunohistochemistry}

Immunohistochemical analysis was performed as described previously [9]. The antibodies were anti-OATP8 (1:100 in 1\% BSA; Santa Cruz, CA, USA) and anti-NTCP (1:1000; Thermo Fisher Scientific) antibodies and analysis was performed at $4{ }^{\circ} \mathrm{C}$ overnight. All slides were examined under an ECLIPSE TE2000-U microscope (Nikon, New York, NY, USA). 


\subsection{Statistical Analyses}

All data were illustrated as the means \pm standard error of the mean (SEM) from at least 3 independent experiments. The Newman-Keuls Test and Dunnett's Multiple Comparison Test were used to determine significance among differences; ${ }^{*} p<0.05$; ${ }^{\#} p<0.01$; and ${ }^{\&} p<0.001$.

\section{Conclusions}

We demonstrated the potential application of NTCP or OATP1B3 and ICG as novel combinations for noninvasive imaging modalities. In addition, OATP1B3 could serve as a dual reporter gene for MR imaging and optical fluorescence imaging.

Supplementary Materials: The following are available online. Figure S1: Genetic engineering of NTCP and OATP1B3. (a) and (b) Western blot analysis NTCP (38kDa) and internal control $\alpha$-tubulin (50kDa) in control and NTCP-expressing HT-29. OATP1B3 $(110 \mathrm{kDa})$ and internal control GAPDH $(37 \mathrm{kDa})$ in control and OATP1B3-expressing HT-29. (c) and (d) Fluorescence microscopy showing the staining for NTCP (green) in (c), OATP1B3 (green) in (d), actin (phalloidin, red), and nuclei (blue). C: control HT-29. N: NTCP-expressing HT-29. O: OATP1B3-expressing HT-29; Figure S2: Proliferation ratio of NTCP and OATP1B3 overexpression. No significant differences were observed. Error bars represent the standard error of the mean. C: control HT-29. N: NTCP-expressing HT-29 cells. O: OATP1B3-expressing HT-29 cells. $\mathrm{N}=4$; Figure S3: Tumor size. (a) NTCP-expressing tumor-bearing mice. (b) OATP1B3-expressing tumor-bearing mice. Tumor sizes were evaluated on day 7,9, 14, 21, and 24 after xenograft. Error bars represent the standard error of the mean. C: control tumor. N: NTCP-expressing HT-29 cells. O: OATP1B3-expressing HT-29 cells; Figure S4: Assessment of FITC intake in vivo in the IVIS. (a) Cells were measured using flow cytometry at the FITC wavelength to observe FITC intensity. (b) The ICG signal was observed using IVIS at 4, 24, 48, and $72 \mathrm{~h}$ after injecting ICG into NTCP- and OATP1B3-expressing tumor-bearing mice. The tumor on the left hind leg was the control tumor, and on the right was the NTCP-expressing tumor (top) and OATP1B3-expressing tumor (bottom). U: untreated. C: control HT-29 cells. N: NTCP-expressing HT-29 cells. O: OATP1B3-expressing HT-29 cells.

Author Contributions: M.-R.W. and J.-K.H. wrote the main manuscript. M.-R.W. performed all analyses (and constructed all figures) except the in vitro MR experiment, which was performed by J.-K.H., Y.-Y.H., and J.-K.H. conducted all experiments.

Funding: Funding was provided by the Tzu Chi Medical Mission Project 105-06-03, Buddhist Tzu Chi Medical Foundation (TCMMP 105-06-03 Buddhist Tzu Chi Medical Foundation) and the National Science Council, and the Taiwan Ministry of Science and Technology (MOST 102-2628-B-303 -001 -MY3).

Acknowledgments: We thank the Taiwan Animal Consortium (MOST 106-2319-B-001-004), which is funded by the Ministry of Science and Technology of Taiwan, for providing IVIS technical support and 7 T Animal MRI Core Lab of the Molecular Imaging Center of National Taiwan University for providing technical and facility support. We also thank the Eighth Core Lab of Department of Medical Research of National Taiwan University Hospital for providing facility support and Hsiao Wan Chi, Lin Yi Ying, and Chen Yi Ru, members of Core Laboratory of the Taipei Tzu Chi Hospital of Buddhist Tzu Chi Medical Foundation for assistance in laboratory studies. This manuscript was edited by Wallace Academic Editing.

Conflicts of Interest: The authors have no conflicts of interest to declare.

\section{References}

1. Comenge, J.; Fragueiro, O.; Sharkey, J.; Taylor, A.; Held, M.; Burton, N.C.; Park, B.K.; Wilm, B.; Murray, P.; Brust, M.; et al. Preventing Plasmon Coupling between Gold Nanorods Improves the Sensitivity of Photoacoustic Detection of Labeled Stem Cells in Vivo. ACS Nano. 2016, 10, 7106-7116. [CrossRef] [PubMed]

2. Dixon, J.E.; Osman, G.; Morris, G.E.; Markides, H.; Rotherham, M.; Bayoussef, Z.; ElHaj, A.J.; Denning, C.; Shakesheff, K.M. Highly efficient delivery of functional cargoes by the synergistic effect of GAG binding motifs and cell-penetrating peptides. Proc. Natl. Acad. Sci. USA 2016, 113, E291-E299. [CrossRef] [PubMed]

3. Sun, N.; Lee, A.; Wu, J.C. Long term non-invasive imaging of embryonic stem cells using reporter genes. Nat. Protoc. 2009, 4, 1192-1201. [CrossRef] [PubMed]

4. Wang, H.; Cao, F.; De, A.; Cao, Y.; Contag, C.; Gambhir, S.S.; Wu, J.C.; Chen, X. Trafficking Mesenchymal Stem Cell Engraftment and Differentiation in Tumor-Bearing Mice by Bioluminescence Imaging. Stem Cells 2009, 27, 1548-1558. [CrossRef] [PubMed]

5. Schönitzer, V.; Haasters, F.; Käsbauer, S.; Ulrich, V.; Mille, E.; Gildehaus, F.J.; Carlsen, J.; Pape, M.; Beck, R.; Delker, A.; et al. In vivo mesenchymal stem cell tracking with PET using the dopamine type 2 receptor and 18F-fallypride. J. Nucl. Med. 2014, 55, 1342-1347. [CrossRef] [PubMed] 
6. Serganova, I.; Ponomarev, V.; Blasberg, R.G. Radionuclide-based reporter gene imaging: Pre-clinical and clinical implementation and application. Nucl. Med. Rev. 2012, 15, 20-36.

7. Deans, A.E.; Wadghiri, Y.Z.; Bernas, L.M.; Yu, X.; Rutt, B.K.; Turnbull, D.H. Cellular MRI Contrast via Coexpression of Transferrin Receptor and Ferritin. Magn. Reson. Med. 2006, 56, 51-59. [CrossRef]

8. Patrick, P.S.; Hammersley, J.; Loizou, L.; Kettunen, M.I.; Rodrigues, T.B.; Hu, D.-E.E.; Tee, S.-S.S.; Hesketh, R.; Lyons, S.K.; Soloviev, D.; et al. Dual-modality gene reporter for in vivo imaging. Proc. Natl. Acad. Sci. USA 2014, 111, 415-420. [CrossRef]

9. Wu, M.-R.; Liu, H.-M.; Lu, C.-W.; Shen, W.-H.; Lin, I.-J.; Liao, L.-W.; Huang, Y.-Y.; Shieh, M.-J.; Hsiao, J.-K. Organic anion-transporting polypeptide $1 \mathrm{~B} 3$ as a dual reporter gene for fluorescence and magnetic resonance imaging. FASEB J. 2018, 32, 1705-1715. [CrossRef]

10. Boni, L.; David, G.; Mangano, A.; Dionigi, G.; Rausei, S.; Spampatti, S.; Cassinotti, E.; Fingerhut, A. Clinical applications of indocyanine green (ICG) enhanced fluorescence in laparoscopic surgery. Surg. Endosc. 2015, 29, 2046-2055. [CrossRef]

11. Antaris, A.L.; Chen, H.; Cheng, K.; Sun, Y.; Hong, G.; Qu, C.; Diao, S.; Deng, Z.; Hu, X.; Zhang, B.; et al. A small-molecule dye for NIR-II imaging. Nat. Mater. 2016, 15, 235-242. [CrossRef]

12. Alander, J.T.; Kaartinen, I.; Laakso, A.; Pätilä, T.; Spillmann, T.; Tuchin, V.V.; Venermo, M.; Välisuo, P. A Review of Indocyanine Green Fluorescent Imaging in Surgery. Int. J. Biomed. Imaging 2012, 2012, 1-26. [CrossRef] [PubMed]

13. Schaafsma, B.E.; Mieog, J.S.D.; Hutteman, M.; van derVorst, J.R.; Kuppen, P.J.K.; Löwik, C.W.G.M.; Frangioni, J.V.; van deVelde, C.J.H.; Vahrmeijer, A.L. The clinical use of indocyanine green as a near-infrared fluorescent contrast agent for image-guided oncologic surgery. J. Surg. Oncol. 2011, 104, 323-332. [CrossRef] [PubMed]

14. James, N.S.; Chen, Y.; Joshi, P.; Ohulchanskyy, T.Y.; Ethirajan, M.; Henary, M.; Strekowsk, L.; Pandey, R.K. Evaluation of polymethine dyes as potential probes for near infrared fluorescence imaging of tumors: Part-1. Theranostics 2013, 3, 692-702. [CrossRef] [PubMed]

15. Luo, S.; Zhang, E.; Su, Y.; Cheng, T.; Shi, C. A review of NIR dyes in cancer targeting and imaging. Biomaterials 2011, 32, 7127-7138. [CrossRef] [PubMed]

16. Guo, J.; Du, C.; Shan, L.; Zhu, H.; Xue, B.; Qian, Z.; Achilefu, S.; Gu, Y. Comparison of near-infrared fluorescent deoxyglucose probes with different dyes for tumor diagnosis in vivo. Contrast Media Mol. Imaging 2012, 7, 289-301. [CrossRef]

17. DeGraaf, W.; Häusler, S.; Heger, M.; van Ginhoven, T.M.; van Cappellen, G.; Bennink, R.J.; Kullak-Ublick, G.A.; Hesselmann, R.; van Gulik, T.M.; Stieger, B. Transporters involved in the hepatic uptake of 99mTc-mebrofenin and indocyanine green. J. Hepatol. 2011, 54, 738-745. [CrossRef]

18. Leonhardt, M.; Keiser, M.; Oswald, S.; Kuhn, J.; Jia, J.; Grube, M.; Kroemer, H.K.; Siegmund, W.; Weitschies, W. Hepatic Uptake of the Magnetic Resonance Imaging Contrast Agent Gd-EOB-DTPA: Role of Human Organic Anion Transporters. Drug Metab. Dispos. 2010, 38, 1024-1028. [CrossRef] [PubMed]

19. Claro da Silva, T.; Polli, J.E.; Swaan, P.W.; da Silva, T.C.; Polli, J.E.; Swaan, P.W. The solute carrier family 10 (SLC10): Beyond bile acid transport. Mol. Aspects Med. 2013, 34, 252-269. [CrossRef] [PubMed]

20. Slijepcevic, D.; Roscam Abbing, R.L.P.; Katafuchi, T.; Blank, A.; Donkers, J.M.; van Hoppe, S.; de Waart, D.R.; Tolenaars, D.; van derMeer, J.H.M.; Wildenberg, M.; et al. Hepatic uptake of conjugated bile acids is mediated by both sodium taurocholate cotransporting polypeptide and organic anion transporting polypeptides and modulated by intestinal sensing of plasma bile acid levels in mice. Hepatology 2017, 66, 1631-1643. [CrossRef] [PubMed]

21. Nkongolo, S.; Ni, Y.; Lempp, F.A.; Kaufman, C.; Lindner, T.; Esser-Nobis, K.; Lohmann, V.; Mier, W.; Mehrle, S.; Urban, S. Cyclosporin A inhibits hepatitis B and hepatitis D virus entry by cyclophilin-independent interference with the NTCP receptor. J. Hepatol. 2014, 60, 723-731. [CrossRef] [PubMed]

22. Yoneya, S.; Saito, T.; Komatsu, Y.; Koyama, I.; Takahashi, K.; Duvoll-Young, J. Binding properties of indocyanine green in human blood. Invest. Ophthalmol. Vis. Sci. 1998, 39, 1286-1290. [PubMed]

23. Wu, M.-R.; Huang, Y.-Y.; Hsiao, J.-K. Role of Sodium Taurocholate Cotransporting Polypeptide as a New Reporter and Drug-Screening Platform: Implications for Preventing Hepatitis B Virus Infections. Mol. Imaging Biol. 2019. Available online: https://www.ncbi.nlm.nih.gov/pubmed/31140111 (accessed on 28 May 2019). [CrossRef] [PubMed] 
24. Shibasaki, Y.; Sakaguchi, T.; Hiraide, T.; Morita, Y.; Suzuki, A.; Baba, S.; Setou, M.; Konno, H. Expression of indocyanine green-related transporters in hepatocellular carcinoma. J. Surg. Res. 2015, 193, 567-576. [CrossRef] [PubMed]

25. Svoboda, M.; Riha, J.; Wlcek, K.; Jaeger, W.; Thalhammer, T. Organic anion transporting polypeptides (OATPs): Regulation of expression and function. Curr. Drug Metab. 2011, 12, 139-153. [CrossRef] [PubMed]

26. König, A.; Döring, B.; Mohr, C.; Geipel, A.; Geyer, J.; Glebe, D. Kinetics of the bile acid transporter and hepatitis B virus receptor $\mathrm{Na}+$ /taurocholate cotransporting polypeptide (NTCP) in hepatocytes. J. Hepatol. 2014, 61, 867-875. [CrossRef] [PubMed]

27. DeBruyn, T.; Fattah, S.; Stieger, B.; Augustijns, P.; Annaert, P. Sodium fluorescein is a probe substrate for hepatic drug transport mediated by OATP1B1 and OATP1B3. J. Pharm. Sci. 2011, 100, 5018-5030. [CrossRef]

28. Tiscornia, G.; Singer, O.; Verma, I.M. Production and purification of lentiviral vectors. Nat. Protoc. 2006, 1, 241-245. [CrossRef]

Sample Availability: Samples of the compounds are not available from the authors.

(C) 2019 by the authors. Licensee MDPI, Basel, Switzerland. This article is an open access article distributed under the terms and conditions of the Creative Commons Attribution (CC BY) license (http://creativecommons.org/licenses/by/4.0/). 